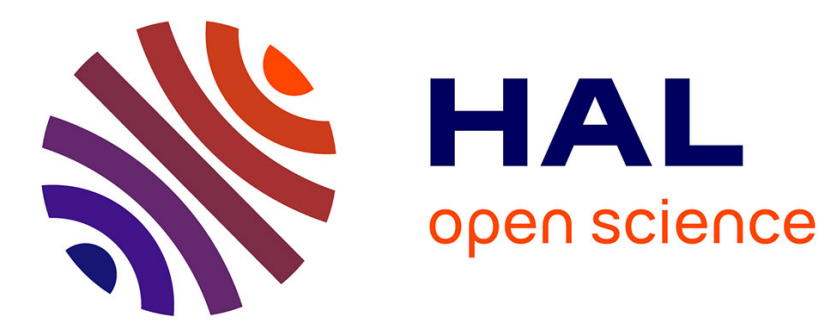

\title{
Adaptation and evaluation of the multiple organs OSD for T2 MRI prostate segmentation
}

Ke Wu, Carole Garnier, Javad Alirezaie, Jean-Louis Dillenseger

\section{To cite this version:}

$\mathrm{Ke} \mathrm{Wu}$, Carole Garnier, Javad Alirezaie, Jean-Louis Dillenseger. Adaptation and evaluation of the multiple organs OSD for T2 MRI prostate segmentation. 36th Annual International Conference of the IEEE Engineering in Medicine and Biology Society, Aug 2014, Chicago, United States. pp.4687-4690. inserm-01059971

\section{HAL Id: inserm-01059971 https://www.hal.inserm.fr/inserm-01059971}

Submitted on 19 Sep 2014

HAL is a multi-disciplinary open access archive for the deposit and dissemination of scientific research documents, whether they are published or not. The documents may come from teaching and research institutions in France or abroad, or from public or private research centers.
L'archive ouverte pluridisciplinaire $\mathbf{H A L}$, est destinée au dépôt et à la diffusion de documents scientifiques de niveau recherche, publiés ou non, émanant des établissements d'enseignement et de recherche français ou étrangers, des laboratoires publics ou privés. 


\title{
Adaptation and evaluation of the multiple organs OSD for T2 MRI prostate segmentation
}

\author{
Ke Wu and Carole Garnier and Javad Alirezaie and Jean-Louis Dillenseger
}

\begin{abstract}
This paper deals with the adaptation, the tuning and the evaluation of the multiple organs Optimal Surface Detection (OSD) algorithm for the T2 MRI prostate segmentation. This algorithm is initialized by first surface approximations of the prostate (obtained after a model adjustment), the bladder (obtained automatically) and the rectum (interactive geometrical model). These three organs are then segmented together in a multiple organs OSD scheme which proposes a competition between the gray level characteristics and some topological and anatomical information of these three organs. This method has been evaluated on the MICCAI Grand Challenge: Prostate MR Image Segmentation (PROMISE) 2012 training dataset.
\end{abstract}

\section{INTRODUCTION}

T2 MRI prostate imaging is now widely used for locating and identifying the prostate cancer by urologists. Beside its diagnostic purpose it allows also to identify targets for prostate biopsy. Prostate MRI also plays an important role in treatment planning (prostatectomy, brachytherapy,...). In our specific case, the medical application is the High Intensity Focused Ultrasound (HIFU) prostate thermal treatment planning and guidance. The segmentation of the external surface of the prostate in T2 MRI has two main purposes: 1) a first external prostate delineation to prepare a future zonal and tumor segmentation for the planning and 2) to extract the information used for a surface/surface preoperative $\mathrm{T} 2$ MRI/peroperative ultrasound (US) registration for the therapy guidance. The delineation of the precise external prostate surface on the T2 MRI is so necessary to ensure an accurate fusion of the preoperative planning into the therapy guidance.

T2 MRI surface prostate segmentation is a challenging problem [1] because of the inhomogeneous image characteristics within and between the several prostatic zones and tumor, of the hyposignal (low gray levels) behavior of the so called prostate capsule, of the links with the neighboring organs and also of the low number of slices describing the prostate. In fact, on T2 MRI, the prostate is generally only acquired on 8-12 slices. The surface of the 2 tips of the prostate, the base and the apex, is parallel to the MR slices and so very badly defined. However, the accurate definition

K. Wu and J.-L. Dillenseger are with INSERM, U1099, Rennes, F35000, France; Université de Rennes 1, LTSI, Rennes, F-35000, France; Laboratory of Image Science and Technology, School of Computer Science and Engineering, Southeast University, Nanjing, 210096, China; and at the Centre de Recherche en Information Biomédicale sino-français, Laboratoire International Associé, co-sponsored by Inserm, Université de Rennes 1, France, Southeast University, Nanjing, China.

C. Garnier is with INSERM, U1099, Rennes, F-35000, France; and Université de Rennes 1, LTSI, Rennes, F-35000, France.

J. Alirezaie is with the Department of Electrical and Computer Engineering, Ryerson University, Toronto, Canada

jean-louis.dillenseger@univ-rennesl.fr of these tips especially the apex is crucial for the therapy. The low quality of the MR in these areas leads to high difficulties for the surface localization, even for the physician.

In an early work, we proposed a T2 MRI prostate surface segmentation scheme based on Optimal Surface Detection (OSD) [2]. Globally, this method gave relatively good results but with some weaknesses, especially near the surrounding organs like the bladder or the rectum because of some false surface definition. The idea is then to use the joint multiple organs segmentation scheme [3] in which the information of the several organs is competing in order to find the more probable surfaces.

In this paper we will first present the anatomical characteristics and segmentation difficulties of the 3 organs. The concurrent segmentation will be described under the OSD framework.

The novelties of this paper are not the OSD or multiple OSD framework themselves (Song for example used the multiple OSD to segment the prostate and bladder on a more simple CT database [3]) but rather the adaptation of these frameworks to our specific case, the way how the user expertise or some anatomical information are introduced in the scheme, the initialization of the 3 organs, the parameter tuning, the quantitative segmentation evaluation on the Promise2012 challenge dataset [1] and some qualitative issues on the final results

\section{Multiple organs Optimal Surface Detection}

\section{A. Optimal Surface Detection principle}

OSD has been originally reported in [4]. The main idea is to refine the surface estimation by a min-cut/max-flow algorithm in the neighborhood of a first surface estimate. This first surface estimate is described as a mesh on which the surface normal $\mathbf{N}_{i}$ on each vertex $v_{i}$ is known (Fig. 1-a). A graph based on this mesh is then built [4], [2].

From each vertex $v_{i}$ a column of nodes is create by the set of the voxels intersected by a ray oriented along the normal $\mathbf{N}_{i}$ and distributed on both side of the surface (Fig.1-b) until a search distance of $\operatorname{In} D(i)$ and $O u t D(i)$, respectively inside and outside the surface.

Intra-column links of infinitive weights connect the nodes of a same column from outside to inside. This construction insures that the final surface intersects the column only once on a node. Inter-columns links of infinitive weights connect nodes of adjacent columns. This links ensure a surface smoothness constraint because the surface can only move from a distance less than $\Delta$ from one column to another 
(Fig.1-b). These 2 sets of links will impose a shape constraint on the surface.
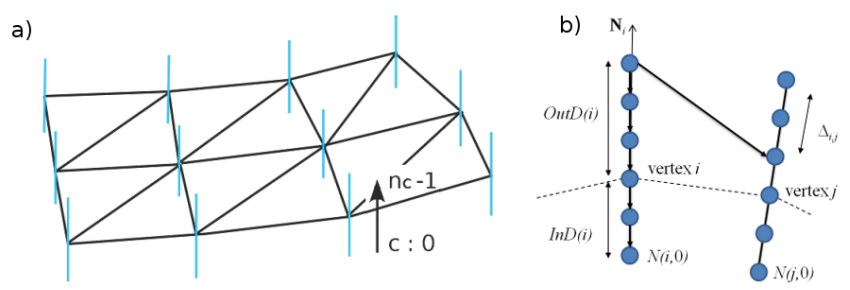

Fig. 1. OSD principle: a) initial surface mesh with the surface normal at the several vertexes; b) graph construction.

Two terminals nodes called $s$ (source) and $t$ (sink) are inserted into the graph. All the nodes of the previous graph will be connected to these two terminal nodes. The weight of the links to the terminal nodes will encode the local imagebased surface energy or the nodes probability to be on the surface. Several weighting functions can be used alone or as a mixture: local gray level gradient based, shape probability based, gradient profile models, etc. (see [4], [2] for more details).

The optimal surface is finally obtained with a mincut/max-flow algorithm [5].

\section{B. Initial prostate segmentation}

The prostate segmentation is a difficult task because in this sequence the prostate gray levels are inhomogeneous inside a prostatic zone and also between the several zones (Fig. 2-a). The prostate is surrounded by a fibromuscular band (the so-called capsule) which presents a low values on T2 MRI. The surface is so define by no-signal. The prostate is only imaged on 8 to 12 axial slices on T2 MRI. Beside the fact that the volume presents a low resolution in the direction perpendicular to the slices, the base and the apex of the prostate are tangent to the slices and so are not well defined (Fig. 2-b). However, a good delineation of these areas is needed for the further treatments.

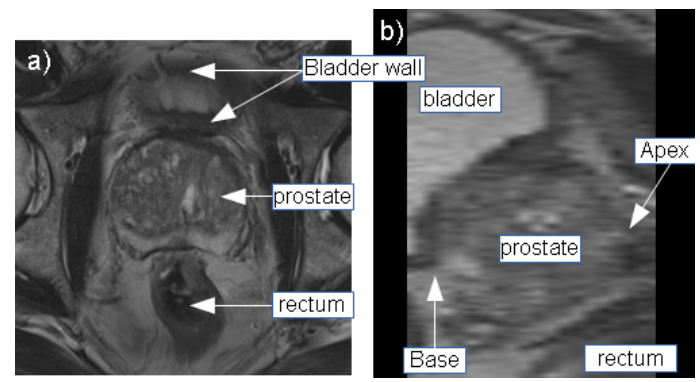

Fig. 2. T2 MRI prostate: a) axial and b) sagittal view.

The OSD segmentation presented in [2] tried to overcome some of these T2 MRI under-determinations. The initialization is performed by an urologist or radiologist who points the apex and the base in the 3D volume. He also positions 4 initial points in a central axial plane. These points introduce expertise within the segmentation process and will serve as anchoring points. An initial mesh is then adjusted on this points by thin plate spline surface. Some specific weights will be given to the links generating from the 6 initial points to constrain the final surface to go over these points positioned by the expert.

\section{Initial bladder segmentation}

The bladder is a distensible organ. Its shape changes continuously according to the amount of collected urine and the position of the surrounding organs. It is placed superior to the prostate base but sometimes can literally envelop the upper part of the prostate. This variability makes it difficult to use a shape model. In T2 MRI, the urine is seen as a high gray level values hypersignal, while the bladder wall itself presents a low gray level values hyposignal with values similar to the prostate capsule (Fig. 2). In 2D, the wall thickness can appear differently on the several slices, especially when the bladder is tangent to the slice as in (Fig. 2-a).

We proposed an initial bladder segmentation framework based on deformable surface [6]. An initial point inside the bladder is located automatically using geometrical (location of the bladder with respect to those of the prostate) and image (high intensities of the urine) features. Centered on this point an initial mesh shaped as a small ellipsoid surface is built. The combination of inflation and internal forces, locally adapted according to the gray levels, allows deforming the mesh toward the bladder inner boundaries while overcoming the leakage issues that can occur at weak edges.

\section{Initial rectum segmentation}

A precise location of the rectum in therapy is absolutely necessary in order to avoid fistula. Near the prostate, the rectum has globally a curved form, but with a wide variability in shape depending on its filling (expansion) and on the position of the surrounding organs. In T2-MRI, if the rectal wall presents a relatively low signal, the materials inside can be totally inhomogeneous in shape and values going from low gray level values (gas) to higher gray level values.

As we did not find automatic methods for robust segmentation of the rectum, we decided to adjust interactively a fast 3D broken tubular model to the data. On an axial slice centered on the prostate, the urologist or radiologist selects two points on both sides of the rectum. These two points give the rectum central tubular section (position and diameter, see Fig. 3-a). The user defines then other two points in the middle axis of the rectum in the front and in the back of the prostate. These two points give the axes directions of the broken tube (see Fig. 3-b)

This model gives a global approximate form of the rectum. However, because of its approximate nature, any specific constraint will be given on the 4 user defined initial point.

\section{E. Multiple organs OSD: initial organs meshes and graph construction}

The initial meshes of the prostate, bladder inner surface and rectum model (see Fig. 3-c) are obtained as described 
previously. In these procedures, the level of interactivity is relatively low: the urologist or radiologist has to select only 6 points for the prostate and 4 points for the rectum model. The interactivity level is so relatively low but absolutely necessary to insert the practitioner expertise into the procedure, especially to define the location of structures badly imaged in T2 MRI like the base and the apex or to model the form of very complex structures like the rectum. However, it can be seen that the 3 meshes can intersect one each other (i.e the rectum and prostate in Fig. 3).

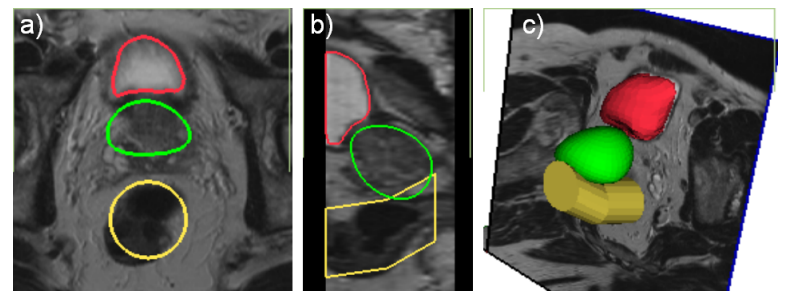

Fig. 3. Initial segmentation of the prostate (green), bladder (red) and rectum (yellow): a) axial, b) sagittal slice and c) 3D shapes.

The next step of the multiple organs OSD is to find the areas where the competition between the organs will be located. For this, each vertex $v_{i_{p}}$ of the prostate surface mesh is projected along the surface normal $\mathbf{N}_{i_{p}}$ toward the bladder and the rectum in order to find correspondent points on these organs $\left(p_{i_{b}}\right.$ and $p_{i_{r}}$ for respectively the bladder and the rectum). The vertex $v_{i_{p}}$ and its correspondent point on the other organs, $p_{i_{b}}$ or $p_{i_{r}}$, will be associated together if the distance between these points is lower than a specified value or if $p_{i_{b}}$ or $p_{i_{r}}$ is inside the prostate surface. The associated points define the competition areas.

A graph is then built for each organ from the 3 initial meshes:

- For the definition of the nodes of each graph, two cases are considered. For the vertexes outside of a competition areas, the column of nodes are performed as described in $\S$ II-A. But inside the competition areas, the column of nodes will incorporate the two associated points and will be distributed on both sides of the 2 surfaces. This will mean that the graphs of 2 different organs will have nodes located at the same position (these in the competition areas).

- For each graph intra- and inter-columns links with infinite weights will be build as described in $\S$ II-A and will have the same surface uniqueness and smoothness purpose.

- Inside the competition areas, as suggested by Song, a surfaces distance constraint is introduced by the incorporation of some infinitive weighted links between the nodes of a column in one organ to the nodes of the corresponding column in the other organ. A specific links topology allows to constrain that the distance between the 2 surfaces in the competition areas lies in a specific interval (see [7] for more detail). This constraint allows to prevent the leakage between surfaces in the interacting area.
- All the nodes will also be connected to the 2 terminal nodes. The main information about the organs will be set as weight on these links:

- Prostate and rectum. We only used as weights the same image-based boundary energy encoding as in [4], [2]. These weights are based on the smoothed gray level gradient of the volume. Because of the inhomogeneity of these organs, other regional gray level information seems difficult to integrate as a weight. However, for the prostate, beside these boundary weights we also introduced the urologist expertise by assigning some arbitrary high weights on the links originating from the 6 initial points nodes.

- Bladder. The initial bladder segmentation give a good estimation of the inner bladder surface wall. Because the bladder wall has a thickness (between $3-5 \mathrm{~mm}$ depending on the filling), we add this information within the graph. All the nodes outside of the initial bladder mesh but closer to the specified wall thickness ( $3 \mathrm{~mm}$ in our case) will be considered as belonging to the bladder. This information will be encoded as weights on the links to the terminal nodes.

The min-cut/max-flow algorithm applied on the common graph allows then to estimate the surfaces of the prostate, bladder and rectum.

\section{RESULTS}

The algorithms were first tested on an own set of $33 \mathrm{~T} 2$ axial MRI volumes from 5 different devices. We had volumes of different qualities and size range from $256 \times 256 \times 24$ to $720 \times 720 \times 20$ voxels with pixel size ranged from 0.391 to $0.781 \mathrm{~mm}$ and axial slice thicknesses from 2.630 to $4.500 \mathrm{~mm}$. On each 33 volumes, an urologist delineated manually the contours of the prostate. These contours have served as ground truth for the segmentation parameters setting or to build the shape model used as the prostate initial model. In a first step, we tuned the several parameters of the OSD framework $(\operatorname{In} D(i)$, OutD $(i)$, Gaussian filter $\sigma$, etc.) in order to maximize the volume overlap between the segmented volume and the ground truth [2]. We kept the best parameters set for the further segmentations.

We choose then to perform the evaluation of the T2-MRI multiple organs on the MICCAI Grand Challenge: Prostate MR Image Segmentation 2012 training dataset [1]. From this dataset, we considered only the 11 T2 MR volumes acquired using an external coil. We discarded the volumes acquired using transrectal coils because the intensity inhomogeneity correction was out of our scope. A manual delineate ground truth was available for all the volumes.

Fig. 4 compares the Dice scores (DSC) of the segmented prostate obtained by the single prostate OSD (Prostate), concurrent prostate and bladder OSD (P\&B) and concurrent prostate, bladder and rectum OSD (P\&B\&R) on each volume. The mean (standard deviation) of the 11 Dice scores is given in Table I. Even if the scores of our methods cannot 


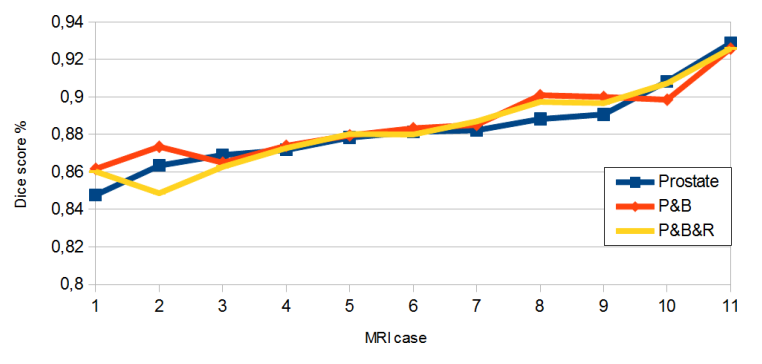

Fig. 4. Comparison of the Dice score obtained by the segmented prostate for the $11 \mathrm{MR}$ volumes. Prostate: single OSD; P\&B: Prostate and bladder joint OSD, P\&B\&R: Prostate, bladder and rectum joint OSD.

be quantitatively compared with these of the best competitor in MICCAI Grand Challenge at that time [8] (we segment only a subset of the challenge dataset), it can be noticed that our methods gave almost the same level of performance.

TABLE I

MEAN AND MEDIAN DSC OF PROMISE TRAINING SET

\begin{tabular}{|c|c|c|}
\hline & Mean DSC & Median DSC \\
\hline \hline Single OSD (P) & $0.8828(0.02)$ & 0.8814 \\
\hline Multiple object OSD (P\&B) & $0.8862(0.02)$ & 0.8833 \\
\hline Mutiple object OSD (P\&B\&R) & $0.8884(0.02)$ & 0.8870 \\
\hline Vincent, et al. [8] & $0.88(0.03)$ & 0.89 \\
\hline
\end{tabular}

The scores do not show statistically significant differences but it seems that multiple organs OSD gives slightly better segmentation scores as the single OSD. However, if we look qualitatively our segmentation results in more details as on Fig. 5, the multiple OSD improves significantly the prostate segmentation. The estimated surfaces are closer to the real topology between the organs. The prostate-bladder OSD segmentation prevents the leakage of the prostate surface to the bladder region (an example is given in Fig. 5-b). The prostate-bladder-rectum OSD segmentation also avoids the overgrowing of the prostate surface in the rectum (Fig. 5-c).
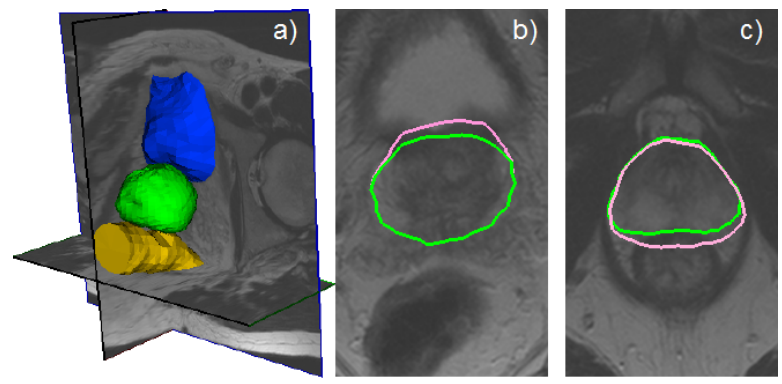

Fig. 5. a) 3D view of the joint prostate, bladder and rectum segmentation. b) and c) zoom on the prostate contour obtained by single OSD (pink) and multiple organs OSD (green).

Fig. 6 gives the DSC of the base, apex and center part of the prostate. The DSC on the center part of prostate is the highest and the most stable in all the 11 cases. But on the apex and base zones, the qualities of segmentation are much lower because the prostate and neighbor organs have there a low resolution and similar intensities. The manual point location will decide the segmentation qualities. In a surgery, the initial points in apex and base zone will be given by an urologist or radiologist with expertise. We have reason to believe that this expertise is helpful to improve the segmentation accuracy in the apex and base areas.

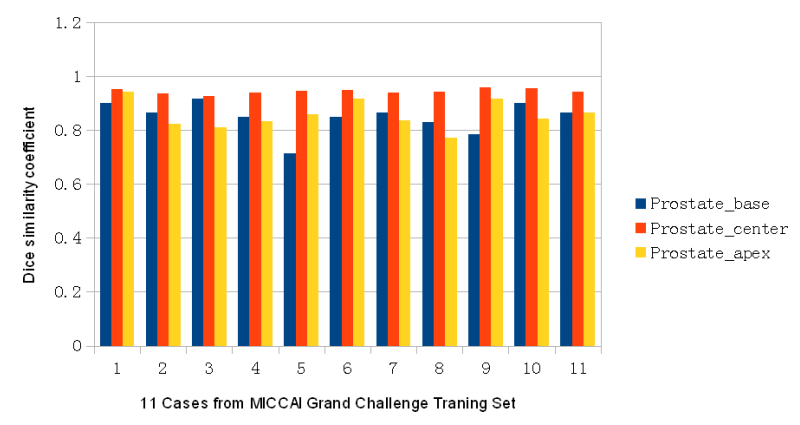

Fig. 6. DSC of the PROMISE training set on different part of the prostate: base (blue), center (red) and apex (yellow)

\section{CONCLUSiON}

In order to improve the prostate segmentation in T2 MRI, we proposed a multiple organs OSD segmentation scheme. This scheme proposes a competitive segmentation between the prostate and the surrounding organs like the bladder and the rectum. This concurrent scheme allowed improving the segmentation by avoiding the estimated prostate surface to cross inside the bladder or the rectum.

\section{ACKNOWLEDGMENT}

This work was part of the MULTIP project (ANR-09TCS-011-04)) and also supported by the National Basic Research Program of China under Grant 2011CB707904, the National Natural Science Foundation of China under Grants 61073138, 60911130370, 61271312, and by a PhD grant from the China Scholarship Council.

\section{REFERENCES}

[1] "MICCAI grand challenge: Prostate MR Image segmentation 2012," http://promise12.grand-challenge.org/, 2012.

[2] K. Wu, C. Garnier, and J.-L. Dillenseger, "Prostate segmentation on T2 MRI using Optimal Surface Detection," IRBM, vol. 34, no. 4-5, pp. 287-290, 2013.

[3] Q. Song, X. Wu, et al., "Optimal graph search segmentation using arc-weighted graph for simultaneous surface detection of bladder and prostate," in MICCAI 2009, London, 2009, pp. 827-835.

[4] K. Li, X. Wu, et al., "Efficient optimal surface detection: theory, implementation, and experimental validation," in Proc. SPIE Med Im, San Diego, 2004, vol. 5370, pp. 620-627.

[5] Y. Boykov and V. Kolmogorov, "An experimental comparison of mincut/max- flow algorithms for energy minimization in vision," IEEE T PAMI, vol. 26, no. 9, pp. 1124-1137, 2004.

[6] C. Garnier, K. Wu, et al., "Bladder segmentation in MRI images using active region growing model," in IEEE EMBS conf, Boston, 2011, pp. 5702-5705.

[7] Q. Song, Y. Liu, et al., "Graph search with appearance and shape information for 3-D prostate and bladder segmentation," in MICCAI, Beijing, 2010, pp. 172-180.

[8] G. Vincent, G. Guillard, and M. Bowes, "Fully automatic segmentation of the prostate using active appearance models," in MICCAI Grand Challenge: Prostate MR Image Segmentation 2012, Nice, 2012. 